agar plates in a slit sampler ${ }^{2}$. Further details of the bactericidal action of vapours of these substances under varying conditions will be published later.

National Institute for Medical Research, J. E. Lovelock. Hampstead, N.W.3.

Nov. 2.

${ }^{1}$ Lovelock, J. E., Lidwell, O. M., and Raymond, W. F., Nature, 153, 20 (1944).

"Bourdillon, R. B., Lidwell, O. M., and Thomas, J. C., J. Hyg., 41, 197 (1941).

\section{Unit Cell and Space-Group of $\mathrm{Cc}_{2} \mathrm{Al}$,}

IN an investigation of cobalt-aluminium alloys by $\mathrm{X}$-ray methods, Bradley and Seager ${ }^{1}$ identified a phase $\mathrm{Co}_{2} \mathrm{Al}_{9}$ or $\mathrm{Co}_{3} \mathrm{Al}_{13}$, thus confirming the findings of earlier workers. The composition of the phase appeared to be invariable, but it could not be fixed exactly in the absence of knowledge of the structure. A phase $\mathrm{FeNiAl}_{3}$ (or $\mathrm{FeNi}_{2} \mathrm{Al}_{13}$ ), found in the ternary system of iron, nickel and aluminium, is isomorphous with the cobalt-aluminium phase, and in a paper on aluminium-rich alloys of the ternary system, Bradley and Taylor ${ }^{2}$ suggest that this phase is monoclinic. The work described below was earried out in order to test this suggestion.

An alloy containing $32 \cdot 7$ per cent cobalt and $67 \cdot 3$ per cent aluminium was made up in a high-frequency induction furnace under a low pressure of hydrogen. The alloy was annealed in vacuo at $900^{\circ} \mathrm{C}$. for 48 hours and allowed to cool in the furnace, after which it was found to be brittle and could be easily crushed. Powder photographs were taken, but it was not found possible to interpret these satisfactorily on the basis of a structure with higher symmetry than monoclinic. Interpretation on the basis of a monoclinic unit cell, that is with four unknown quantities, is not practicable. It was therefore decided to try to isolate single crystals of the phase, so that the unit cell could be obtained directly from oscillation and Weissenberg X-ray photographs.

An examination of the crushed fragments under a microscope showed a small percentage of regularly shaped pieces. Some of these were mounted with shellac on glass fibres and photographed in an X-ray camera of $2 \cdot 8 \mathrm{~cm}$. diameter specially designed to reduce exposure time in these preliminary experiments ${ }^{3}$. The photographs so taken usually contained an irregular distribution of spots, showing that the fragment consisted of a number of crystals, but sometimes traces of layer lines could be detected. From the orientation of these layer lines the crystal could be set with one axis parallel to the axis of oscillation. When this was achieved it was often found that other crystals were also present, giving spots lying between the layer lines. Finally one fragment was found which gave layer lines with no spots between them; this was then known to be a single crystal.

From the single crystal obtained in this manner, the unit cell was found to be monoclinic with $a=6 \cdot 18 \pm 0.02 \mathrm{kX} ., b=6 \cdot 24 \pm 0.02 \mathrm{kX} ., c=$ $8.59 \pm 0.02 \mathrm{kX} ., \beta=95^{\circ} \pm 0.5^{\circ}$. With these para meters it was possible to index the lines on the powder photograph, thus showing that the fragment selected was of the same phase as the bulk of the material. From the unit cell dimensions combined with density measurements it is found that there are four cobalt and eighteen aluminium atoms per unit cell; the density calculated on this basis is 3.62 gm./c.c., which agrees with the observed density of $3.673 \mathrm{gm}$./c.c. Thus the composition is represented by the formula $\mathrm{Co}_{2} \mathrm{Al}_{9}$. The space-group is $P 2_{1} / c$ which has four general equivalent positions per unit cell. Hence two of the aluminium atoms must lie on special positions on one of the four sets of centres of symmetry, but it is probable that all the other atoms are in general positions.

Further work on the complete determination of the structure is in progress.

\section{Cavendish Laboratory, Cambridge. Sept. 12.}

1 Bradley, A. J., and Seager, G. C., J. Inst. Met., 64, 81 (1939). ${ }^{2}$ Bradley, A. J., and Taylor, A., J. Inst. Met., 66, 53 (1940). 'Parker, A. M. B., J. Sci. Instruments, 22, 131 (1945).

\section{A New Test for $2 \times 2$ Tables}

MAY I reply briefly to some of Prof. R. A. Fisher's remarks ${ }^{1}$ on my suggested test ${ }^{2}$ ?

First, Prof. E. B. Wilson ${ }^{3}$ was concerned with tables in which all marginal totals were equal ( $m=n=r=s$ in my notation). He calculated the distribution of the difference $(a-b)$ on the assumption that $p$ was given by the column totals to be $\frac{1}{2}$; and his test was based on this distribution. Apart from the facts $(a)$ that my proposal involves neither $m=n$ nor $r=s$, and $(b)$ that my test is not based on the difference $(a-b)$, there is an essential difference of principle between my proposal and that of Prof. Wilson, in that he assumes $p$ to be given by the column totals, while I make no such assumption. My validity condition explicitly covers all possible values of $p$.

Prof. Fisher's criticism ${ }^{4}$ of Prof. Wilson was based primarily on this assumption about the unknown $p$, and it seems a valid criticism of this assumption; but my proposal is unaffected by it.

Secondly, I wish to emphasize that I do not believe that the conditions stated in my earlier letter for the validity of the test I proposed are always satisfied in practice. If they were, there would have been little point in stating them. Conditions can arise where it is altogether false to assume $p$ constant. In such circumstances, we can only try to randomize beforehand ; and then, if this is done, Prof. Fisher's test remains valid - a fact not adways realized (for example, Wald ${ }^{5}$.

'Thirdly, concerning Prof. Fisher's remark that the result where all animals die is irrelevant to the null hypothesis, it may be that this difference of opinion results from a difference of attitude. Suppose we consider two 'null hypotheses' : (1) blue-eyed people are just as likely to catch colds as non-blue-eyed people; (2) taking a daily dose of $X Y Z$ does not affect the chance of having a cold. Each of these hypotheses might be tested by an experiment yielding a $2 \times 2$ table. But there is a difference of attitude. In case (1), we do not have in mind any proposal to do away with blue-eyed people in order to reduce colds ; in case (2) we do have in mind giving people $X Y Z$, if it should turn out to be good. In case (1) we study the world; in case (2) we propose to change it. Our conceptual universe in case (1) is a single fourfold one, in which a given individual can be classified by two distinet attributes-'blue-eyed' and 'cold'. In case (2) we have in mind two distinct two-fold wòrlds ; one possible world, in which everybody has $X Y Z$ 